\title{
Meta
}

Journal des traducteurs

Translators' Journal

\section{Oittinen, R. (2000) : Translating for Children, New York and London, Garland Publishing, 205 p.}

\section{Louise Audet}

Volume 48, numéro 4, décembre 2003

URI : https://id.erudit.org/iderudit/008741ar

DOI : https://doi.org/10.7202/008741ar

Aller au sommaire du numéro

Éditeur(s)

Les Presses de l'Université de Montréal

ISSN

0026-0452 (imprimé)

1492-1421 (numérique)

Découvrir la revue

Citer ce compte rendu

Audet, L. (2003). Compte rendu de [Oittinen, R. (2000) : Translating for Children, New York and London, Garland Publishing, 205 p.] Meta, 48(4), 617-627.

https://doi.org/10.7202/008741ar

Ce document est protégé par la loi sur le droit d'auteur. L'utilisation des services d'Érudit (y compris la reproduction) est assujettie à sa politique d'utilisation que vous pouvez consulter en ligne.

https://apropos.erudit.org/fr/usagers/politique-dutilisation/
Cet article est diffusé et préservé par Érudit.

Érudit est un consortium interuniversitaire sans but lucratif composé de l'Université de Montréal, l'Université Laval et l'Université du Québec à Montréal. Il a pour mission la promotion et la valorisation de la recherche. https://www.erudit.org/fr/ 
modifications subies par l'œuvre, elle utilise aussi les brouillons de deux conférences données par le traducteur à l'occasion de premières de la pièce. Enfin, Anna Soler s'attache à la réception du théâtre de G. B. Shaw en Catalogne de 1908 à 1938; pour ce faire, elle rend compte des différentes mises en scène, des traductions et des réactions de la critique. Elle conclut que les attentes de renouveau du canon esthétique par des modèles créatifs, nourries par les intellectuels qui ont "importé» le théâtre de Shaw, n'ont pas trouvé l'écho espéré.

Les trois travaux suivants portent sur les poètes symbolistes. Le premier, de Pilar Gómez Bedate, s'attarde à la très importante anthologie intitulée La poesía francesa moderna et compilée par Díez Canedo et Fortún en 1913 (puis revue et augmentée en 1945). Les circonstances de l'élaboration de cette anthologie, qui a marqué l'évolution de la poésie espagnole, sont décrites et plusieurs poèmes analysés. Le deuxième, de Soledad González, étudie les premières traductions de Verlaine par Juan Ramón Jiménez et plus précisément les révisions introduites par le traducteur au fil des années. Le dernier, de Francisco Ruiz Casanova, s'attelle à retracer les traductions dans les pages de la revue madrilène La República de las letras et avance certaines hypothèses au changement de politique éditoriale de la poésie à la prose.

"Las Vanguardias», les Avant-gardistes, est le sujet des deux articles de ce cinquième bloc. Miguel Gallego Roca, en fait, nous explique pourquoi les auteurs d'avant-garde européens n'ont pas vraiment été traduits en Espagne. Traduire et publier le Manifeste futuriste de Marinetti ne signifie pas nécessairement une poétique avant-gardiste qui recherche de nouveaux horizons linguistiques et formels au moyen de la traduction. Tout le contraire, selon Gallego Roca, ces traductions se rapprochent davantage d'une "poétique symboliste traduite selon un code moderniste hispanique» (p. 42). La poésie importée n'est donc pas provocation, mais bien réaffirmation des conventions. Dans le deuxième article, Alicia Piquer nous parle de Jorge Guillén, traducteur de Supervielle, et comment ses traductions établissent une véritable relation intertextuelle avec sa production originale.

Finalement, un travail centré sur le personnage du traducteur: Teodoro Llorente. Francisco Lafarga analyse minutieusement les circonstances de la composition de l'anthologie Poetas franceses del siglo XIX (1906), qui comprend 376 poèmes traduits par Llorente, le travail du traducteur et la réception de l'anthologie.

Dix-neuf études qui renseignent le lecteur sur le rôle de la traduction et des traducteurs au cours de l'âge d'argent, qui éclairent l'évolution de la littérature espagnole et catalane moderne. Traduction et littérature comparée s'y rencontrent à nouveau.

GeORges L. BAstin

Université de Montréal, Montréal, Canada

Oittinen, R. (2000): Translating for Children, New York and London, Garland Publishing, $205 \mathrm{p}$.

«Quand la fonction prend le pas sur le texte...»

Le présent ouvrage s'inscrit dans la collection portant sur la littérature et la culture pour enfants dont l'objet est de promouvoir la recherche dans ce domaine et de tracer de nouvelles perspectives grâce à la collaboration de chercheurs du monde entier.

Riitta Oittinen, professeur de traduction à l'Université de Tampere (Finlande), s'intéresse aux questions de sémiotique. Elle est également illustratrice et productrice de dessins animés pour la jeunesse. Comme elle le rappelle dans sa préface, la traduction a joué un rôle vital en Finlande dans l'affirmation culturelle et politique de cette petite nation isolée linguistiquement et coincée entre ses puissants voisins scandinaves et slaves. Cependant, malgré la riche tradition de traduction de la littérature enfantine dont jouit la Finlande, ce 
domaine avait fait l'objet de peu de recherches traductologiques jusqu'au début des années 1980. Avec son livre, Riitta Oittinen vient combler une lacune et démontrer la vigueur des recherches de traductologie en Finlande ainsi que l'intérêt croissant pour les recherches dans ce domaine.

L'auteur propose ici une étude et une réflexion axées sur le rôle du traducteur, charnière essentielle de la communication translinguistique qui s'établit entre auteurs et enfants lecteurs. Elle fonde son analyse sur le concept du dialogisme, en référence aux travaux de Mikhaïl Bakhtine, auquel elle adjoint le principe de loyauté, élaboré par l'approche fonctionnaliste en traduction (Christiane Nord) et les théories sociologiques visant à réhabiliter le rôle du traducteur sur les plans social et littéraire (Robinson). Ainsi, elle se démarque nettement des approches traditionnelles basées sur les notions d'équivalence, de fidélité entre textes, du vouloir-dire de l'auteur, tel qu'il se manifeste dans le texte. Pour elle, ces approches relèguent le rôle du traducteur dans l'oubli:

The translator's centered approach to the study of translation differs sharply from older, more traditional approaches that are focused on abstract structures of equivalence, «matches», or «fidelities" between texts (in words). Thus, I do not agree with views that see translation as a mechanistic act pertaining to texts as such, to the author's intentions and issue of language. In this way, the translator's action is relegated to obscurity, if not invisibility (p. 3).

L'objectif de l'étude présentée dans l'ouvrage est double. L'auteur entend, d'une part, mettre en lumière le rôle du traducteur, particulièrement dans le cas de la traduction pour enfants, et, d'autre part, démontrer la primauté du contexte global (la situation historique, cultu-

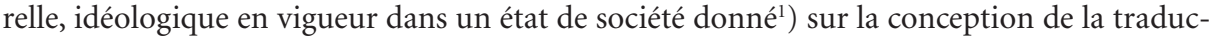
tion comme recherche et reproduction des intentions de l'auteur telles qu'inscrites dans le texte. Posant le principe de la loyauté du traducteur comme seul garant de fidélité, elle s'insurge contre la conception de la traduction «transparente» qui ne ferait que restituer dans une autre langue l'exact pendant du texte source. C'est, en effet, en affirmant son rôle déterminant, en se rendant visible, en orientant son travail vers le lecteur récepteur, que le traducteur fera preuve de la plus grande fidélité envers l'œuvre:

[...] I am concentrating on human action in translation, and I hope to shed some light on the translator, the translation process, and translating for children, in particular. My intention is to demonstrate how the whole situation of translation takes precedence over any efforts to discover and reproduce the original author's intentions as a given. Rather than the authority of the author, I focus special attention on the intentions of the readers of a book in translation, both the translator and the target-language readers (p. 3).

La littérature pour enfants est ainsi définie: «I see children's literature as literature read silently by children and aloud to children » (p. 4). L'auteur précise cette première définition, implicite, généralement acceptée par les auteurs et les éditeurs en relevant deux caractéristiques fondamentales de ce champ littéraire dont, selon elle, les chercheurs ont peu tenu compte. La première est constituée par les illustrations:

Illustrations are of major importance in children's literature, especially in books written for illiterate children. The illustrations in picture books may often be even more important than the words, and sometimes there are no words at all. Illustrations have also been of little interest for scholars of translation, and there is hardly any research on this issue in translation studies» (p. 5).

La seconde relève du fait que ces œuvres sont conçues pour être lues à voix haute: «Reading aloud, too, is characteristic of books for children» (p. 5).

L'ouvrage prendra en compte et approfondira ces deux aspects fondamentaux de la traduction pour enfants: la lecture (à voix haute ou silencieuse) ainsi que la mise en relation des mots avec les illustrations.

Le livre est organisé en six chapitres. L'auteur, après avoir défini ses objectifs ainsi que le choix de son approche, aborde les questions centrales de la situation contextuelle et de 
l'équivalence (chapitre 1). À l'instar des théoriciens des écoles fonctionnaliste (Nord, Reiss et Vermeer) et sociologique («manipulative school», p. 10), elle souligne le caractère communicatif de la traduction dont les principaux agents sont, nous rappelle-t-elle, des personnes (auteur, traducteur et lecteurs) ayant prise sur les événements (processus) et les objets (textes):

As I see it, it is far too often that we neglect the function of the different human beings in a translation situation. Texts do not function withour human beings. Thus the function of a text is not « as such» but is redefined every time the text is read. A text in translation is influenced by the author, the translator, and by the expectations of the target-text readers (p. 12).

Elle consacre le chapitre deux à l'aspect interprétatif de la lecture-traduction. La pierre d'assise d'une théorie de la traduction pour enfants repose - dit l'auteur - sur la compréhension et la lecture qu'elle définit comme une transaction, une situation dialogique et une performance. Événements uniques dans le temps, chaque fois renouvelés et différemment perçus par les acteurs. Le chapitre trois traite de la problématique du lecteur enfantin. Si, comme le postule l'auteur, la traduction se définit comme une communication, alors, traduire pour les enfants c'est établir une communication entre les adultes et les enfants. D'où l'importance de la conception de l'enfant dont devraient toujours tenir compte les (re)créateurs:

[...] our concepts of childhood are mirrored in every adult act, in all creations for children. When we write, illustrate, or translate for children, we always do it on the basis of our images of childhood, on the basis of the whole society's image of childhood. When we create for children, we have a certain kind of childhood and children in mind. When we censor, what and how we do so is based on our child concept (p. 53).

À cet égard, Oittinen reconnaît dans la définition de l'enfant d'Alice Miller ${ }^{2}$ une conception très proche de la sienne: un enfant qu'il faut respecter et écouter, un être capable d'effectuer des choix.

Les différences psychologiques de ces récepteurs contribuent à définir le caractère spécifique de la culture enfantine qui, telle la culture carnavalesque de Rabelais, ne se définit pas en opposition avec la culture des adultes, mais à l'extérieur d'elle. Pour Oittinen, cette comparaison offre un point de vue nouveau sur la culture enfantine tout en permettant de reconnaitre la valeur d'une autre approche que celle des adultes: une communication non autoritaire, dialogique, véritable rencontre ludique d'un «je» et d'un «tu». D'emblée, l'invitation à entrer dans le jeu est lancée:

As a whole, children's culture could well be seen as one form of carnivalism - imagine the situation where we as translators for children, join the children and dive into their carnival, not teaching them but learning from them. Through and within dialogue, we may find fresh new interpretations, which does not mean distortion, but respect for the original, along with respect for ourselves and for the carnivalistic world of children (p. 58).

D'ailleurs, Riitta Oittinen préfère désigner ce champ littéraire sous l'étiquette de littérature pour enfants plutôt que de littérature enfantine ou de littérature d'enfance (chapitre 4). L'auteur préfère même éviter toute définition trop explicite de cet objet d'étude:

On the whole, because I consider children's literature a dialogic event, rather than "an object or a thing in itself", it would be difficult, to substantiate any stabilized function or stabilized meaning. Thus I have tried to avoid giving explicit definitions of children's literature. In a wide sense, children's literature can be seen as anything children read. Here, it could mean books published for children by adults (p. 69).

Outre l'aspect dialogique de la traduction, la traduction pour les enfants est caractérisée par le rôle majeur accordé au traducteur qui, conformément à l'approche fonctionnaliste, devient le pivot central de l'acte traductif: c'est lui qui, en affichant sa loyauté, non seulement envers les lecteurs du texte traduit, mais envers sa propre conception de l'enfant, assure le succès de 
la traduction dont la mesure en est l'effet produit sur l'enfant lecteur. Ainsi, l'auteur privilégie une conception dynamique et fonctionnelle de la traduction:

Moreover I prefer to speak of translating for children instead of the translation of children's literature as translators are always translating for somebody and for some purpose; translators are not just replacing old things with new ones. Translating for children rather refers to translating for a certain audience and respecting this audience through taking the audience's will and abilities into consideration. Here the translator's is a crucial factor (p. 69).

Le chapitre cinq traite longuement des questions relatives au statut du texte original par rapport au texte traduit et à l'adaptation (intra- ou interlinguistique, picturale ou musicale). L'auteur illustre ses thèses par l'analyse de traductions et d'adaptations (pragmatiques, linguistiques et sémiotiques) d'œuvres, effectuées en fonction des divers horizons de réception. Elle en propose une étude non normative, dont la visée est centrée sur la compréhension des processus mis en œuvre lors de la traduction pour enfants: comment s'effectue cette communication avec les enfants par la traduction. La traduction est ainsi envisagée comme une communication interculturelle ou transculturelle (culture des adultes et culture enfantine), ouverte et toujours renouvelée.

Dans la première partie de ce chapitre, l'auteur remet en question les concepts d'altérité et de visibilité tels que préconisés par Berman et Venuti. Pour elle, cette pratique "éthique» ne prend pas assez en considération l'importance de la problématique de la réception: la diversité des destinataires ainsi que leurs réactions. Ce n'est pas en faisant pénétrer l'étrangéité du texte source dans la culture cible que le traducteur se révèle, mais au contraire, soutient Oittinen, en adaptant et en domestiquant le texte source qu'il se rend visible:

Venuti does not address the complexities concerning the multiplicity of readers and reader's response. [...] While interpreting stories and rewriting them for future readers, translators are acting on the basis of their own child images, which means that while adaptating, they are in the end more visible than invisible (p. 74).

Elle se range aux côtés de Nord et de Robinson, pour qui le critère de correction n'est pas établi en fonction du texte original, mais bien de la lecture de l'original par le traducteur. Ainsi, les notions de traduction et d'adaptation relèvent davantage de questions d'attitude que de la structure même des œuvres.

In my view, the main difference between translation and adaptation lies in our attitudes and points of views, not in any concrete difference between the two. As Robinson would have it, the translator's correction is not in the original, but in the translator's reading of the original; in other words, translation is not an issue of unity but of recreation and deviation, «turning» the original «into a new and unexpected form» (p. 80).

D'ailleurs, ajoute-t-elle, Steiner ne voit-il pas l'adaptation comme un moyen d'assurer la pérennité des œuvres?

Elle concède, avec Zohar Shavit, que la question de l'adaptation est souvent liée au statut de la littérature pour enfants et que c'est avec raison que certains auteurs, comme Birgit Stolt et Astrid Lindgren, veulent assurer aux récepteurs de littérature pour enfants des traductions d'aussi bonne qualité qu'aux adultes. Ce souci est justifié dans la mesure où, au cours des siècles, les œuvres ont souvent été adaptées en fonction des principes pédagogiques des adultes, aux dépens des goûts et des besoins des enfants. Cependant, note Oittinen, ces théoriciennes, en critiquant les pratiques d'adaptation, de manipulation des œuvres de la littérature d'enfance, prennent comme référence le texte original, en tant que forme et contenu, objet stabilisé, concepts qu'elle-même juge trop vagues:

I share Shavit's and Stolt's worry about the status of children literature and I understand their point, but I can only agree in part with their views about the translation of children's literature. [...] Yet both the «meaning» and the «text» are vague concepts: as pointed out earlier, texts and meanings never exist as such, they are always interpreted and reinterpreted in a situation (p. 81). 
De plus, commente l'auteur, il est pratiquement impossible de satisfaire à l'exigence d'un psychologue comme Bettelheim qui souligne l'importance, pour la psychè enfantine, de recourir à la lecture de contes dans leur version originale. Pour Oittinen, il est illusoire de penser remonter au texte fondateur. Le fait que Bakhtine ait relevé de nombreuses similarités entre l'histoire d'Alice, les fables d'Orphée et l'Âne d'or d'Apulée fait-il du conte de Carroll une adaptation? Pour elle, tenter de distinguer la part de l'original, de celle de l'adaptation ou de la traduction est plus problématique qu'éclairant: elle ne voit pas de différence méthodologique entre l'adaptation et la traduction, l'important étant d'assurer le bon fonctionnement de la traduction dans le contexte cible:

In general, if we try to define adaptation and translation as separate issues, we face a dilemma, as we are actually mixing terms on different levels: when translating, we are always adapting our texts for certain purposes and certain readers, both children and adults. The translation process as such bring the text closer to the target-language readers by speaking a familiar language. Domestication is part of translation, and not a parallel process. There is no real methodological difference between the two. What really matters here, is how well translations function in real situations, where the «I» of the reader of the translation meets the «you» of the translator, the author, and the illustrator (p. 84).

La deuxième partie de ce chapitre, "Breaking the Closed System", est consacrée aux questions relatives à la traduction et à l'adaptation de la littérature pour les enfants. L'auteur retient les travaux de deux spécialistes Zohar Shavit ${ }^{3}$ et Göte Klingberg ${ }^{4}$ qui ont traité du statut de la littérature pour enfants. Elle considère cependant que ces auteurs ont davantage orienté leurs études sur ce que, en termes bakhtiniens, elle nomme le «donné» (langage, culture, contexte historique), plutôt que le «créé» (interprétation, transformation, création). Ainsi, bien qu'elle ait su montrer l'importance des facteurs historique, culturel et géographique sur les différentes représentations de l'enfant, Shavit a négligé de mettre en relief l'influence jouée par le contexte individuel à la réception, celui des traducteurs et des enfants lecteurs. Quant à Klingberg, il opte pour une approche plus didactique, fondée sur l'analyse contrastive d'extraits de textes, avec l'objectif d'établir des règles strictes pour les traducteurs. Il établit de nettes distinctions entre les notions d'adaptation, de versions abrégées de l'œuvre originale (admissibles lorsqu'elles sont autorisées par l'auteur et non par le traducteur). Il critique au passage les interventions "protectionnistes » au niveau des textes et des illustrations: purification, assainissement des valeurs (par omission et addition) et modernisation (adaptation linguistique contemporaine). Cette critique trahit, selon Oittinen, une conception de la traduction trop orientée vers la reproduction du texte source, qui nie l'autonomie du traducteur et du lecteur. Un exemple intéressant de purification est celui de la censure relative à certains tabous (l'alcool, la mort, la sexualité, le scatologique) relevés par les théoriciens. Oittinen cite le cas de deux traductions en finnois de l'œuvre de Jonathan Swift, Gulliver's Travels, dont l'une a été effectuée à partir de la version abrégée de l'original. Il est intéressant de constater, note-t-elle, combien les passages omis (violence, excréments, non respect envers les adultes) correspondent aux cas de censure relevés par Shavit dans des traductions en hébreu de cette histoire. Là où, dans le texte original, Gulliver éteint un feu avec son urine (parce qu'il avait bu du vin), la version finnoise mentionne de l'eau, et l'une des versions en hébreu, son souffle. Les passages relatifs à la défécation sont soit traduits de façons différentes, soit omis. Dans ces cas, pour respecter l'esprit carnavalesque (rabelaisien) qui caractérise la culture enfantine, Oittinen se prononce clairement contre cette forme d'adaptation et de censure:

The adaptor (and/or publisher) probably felt that it was not appropriate to mention defecation in a children's story. Here the adaptor has "not dived into children's carnivalism », but she has looked down on children, censoring their experience of literature. I could imagine a child's interest in Gulliver's problems: if you are a giant, and everybody else is small, urinating and defecating are certainly something you need to worry about (p. 93). 
Par ailleurs, tout en concédant avec Klinberg que les enfants doivent pouvoir jouir de traductions de haute qualité, elle critique sa conception de l'erreur. Elle cite à l'appui le cas de deux traductions finlandaises des Aventures d'Alice: alors que Lewis Carroll décrit la rencontre de son héroïne avec une chenille bleue, les deux traductions mentionnent une chenille verte. Si, pour Klingberg, il s'agit d'une erreur, il en est autrement pour Oittinen. Même si, comme dans l'édition la plus récente, les illustrations en couleurs de Tove Jansson, effectuées d'après le texte original, représentent une chenille bleue! Cette disparité n'en provoquera que davantage l'étonnement et la participation de la part de l'enfant lecteur, assure l'auteur, après avoir lu l'histoire à ses propres enfants.

Finalement, conclut Oittinen, Shavit et Klinberg se leurrent en considérant l'adaptation comme une solution négative, une manifestation de manque de respect envers les enfants. Toute traduction entraîne nécessairement une adaptation.

La troisième partie porte sur les aspects extralinguistiques de la traduction tels que les illustrations, la musique, le théâtre. L'auteur insiste sur un point des plus importants pour elle: les traducteurs de livres pour enfants traduisent plus que des textes en mots. Ils doivent également tenir compte de l'ensemble de l'œuvre, c'est-à-dire les mots (y compris la typographie), les illustrations, le contexte de la lecture à voix haute. Tous ces aspects constituent pour elle autant de formes de traductions et sont traités dans le cadre général de la théorie dialogique bakhtinienne.

Through the examples in the third section, I also aim to show in practice how important it is for translators to translate more than texts in words. Translators of picture books translate whole situations including the words, the illustrations, and the whole (imagined) reading-aloud situation. Illustration is a many-faceted phenomenon in translation: on the one hand, illustrations go along with translations and their originals; on the other hand, illustrations can be understood as a form of translations as such. Yet I have deliberately avoided relying on any separate theory of illustration and have dealt with illustration within the framework of translation, as part of the Bakhtinian dialogics of translation (p. 75).

La complexité même du processus soumet les traducteurs à des exigences spécifiques: la traduction pour les enfants constitue un champ de spécialisation. En effet, la présence des illustrations crée une nouvelle exigence pour le traducteur: d'abord, il doit associer le texte avec les images et, consciemment ou inconsciemment, il intègre à ce processus son propre imaginaire. Pour assurer le succès de la traduction, le traducteur doit non seulement tenir compte de chacun des éléments constitutifs de l'œuvre - les mots, les sons, les mouvements, les illustrations - mais être en mesure de les interpréter. Il doit en outre tenir compte de l'interaction de l'enfant lecteur avec les illustrations. Ici, Oittinen admet que la traduction de livres illustrés constitue un champ de spécialisation des études de traduction qui requiert une formation intégrant, par exemple, des connaissances en art.

Dans la cinquième partie du chapitre, Oittinen aborde la question de l'auteur-traducteur. Remettant en question le statut de la «primauté» de l'auteur par rapport à son œuvre, elle rejette l'opinion d'un Robert de Beaugrande à propos de la reconnaissance des «droits» de l'auteur, seul habilité à modifier et à réinterpréter son œuvre. C'est pour elle une attitude élitiste. D'ailleurs Bakhtine, dans sa description de la tâche et du rôle de l'auteur-créateur et du lecteur-créateur, ne reconnaît-il pas l'influence du contexte historique, culturel et géographique sur la création? Ainsi, conclut Oittinen, la traduction d'une œuvre par son propre auteur n'apporte aucune garantie quant à la fidélité, ne serait-ce qu'en raison du nouveau contexte historique. Elle invoque également les travaux de Zohar Shavit sur la comparaison de versions par Roald Dahl de ses œuvres pour les enfants. Les versions pour les enfants diffèrent sous plusieurs aspects: la langue, la structure narrative, l'ordre chronologique, la conclusion de l'histoire, en général plus complexes et ambigus dans la version pour adultes.

Les exemples analysés par Oittinen confirment cette tendance : la comparaison des versions et adaptations de leurs propres œuvres par Tove Jansson et Lewis Carroll révèle des 
différences (ton, normes, langue, structure et la conclusion), selon qu'elles s'adressent à un public adulte ou jeunesse.

These examples clearly demonstrate the close connection between translation and adaptation; they are both forms of rewriting, editing, and collaboration, and drawing an absolute distinction between the two is quite difficult (p. 75).

La dernière partie est consacrée à l'étude comparative de trois versions en finnois d'Alice's Adventures in Wonderland, selon deux paramètres: l'historicité et la conception de l'enfant. La première traduction est de Anni Swan (1906), la seconde, de Kirsi Kunnas et Eeva-Liisa Manner (1972) et la plus récente, par Alice Martin, a paru en 1995. Pour l'auteur, ces traductions sont tributaires de leurs époques par leurs objectifs et les images de l'enfance dont elles sont porteuses. Ces caractéristiques sont manifestes au niveau des stratégies de traduction, des récepteurs ainsi que des points de vue différents sur l'histoire. Swan et Kunnas ont «domestiqué» l'histoire afin de la rendre plus accessible aux jeunes Finlandais, mais Martin a adopté une autre approche: elle n'a pas craint de confronter ses lecteurs à l'étrangéité du texte source. Selon Oittinen, cette différence d'approche s'explique par les changements sociaux et politiques qu'a connus la Finlande, surtout depuis son entrée dans l'Union européenne en 1995. Le pays n'est plus aussi isolé qu'il l'était et, grâce aux progrès technologiques (comme Internet), plus ouvert à l'influence anglo-saxonne:

Even if our knowledge of the world may still not be sufficient, we have learned to tolerate otherness more than we did before. Again, this shows in the translations published. For instance, names no longer need to be translated. Unlike the 1906 and 1972 versions, where Alice is called «Liisa», the 1995 version lets Alice keep her British name (p. 139).

Dans sa conclusion, l'auteur rappelle les multiples facteurs sociologiques et historiques dont est tributaire l'acte traductif (le statut de l'œuvre source en regard du statut des traductions-adaptations) et elle appelle à la collaboration entre les agents (auteurs, traducteurs, éditeurs, lecteurs) de cette communication sans cesse renouvelée. L'élément privilégié de la traduction de la littérature pour enfants reste avant tout son récepteur: l'enfant imaginaire et l'enfant récepteur du texte traduit:

When translating for children, we should listen to the child, the child in the neighborhood and the child within ourselves. When reading and writing, authoring and illustrating, the translator is in a dialogic interaction with all these children. A thought, a sentence, a text, a picture - they are all involved in a never-ending dialogue. They are continuously changing, moving, and they never meet in a vacuum. In different reading situations, readers interpret theses signs in various ways, depending on the situation itself: text, interpreter, time, place and so on. Form and content are inseparable (p. 168).

De la caractérisation de ce champ littéraire à celui de ses récepteurs (l'enfant-lecteur et l'adulte), en passant par les diverses modalités d'expression mises en jeu (oralité, illustrations, adaptations transculturelles, transsémiotiques), l'on voit à quel point le champ est aussi exigeant que passionnant.

Nous ne saurions remettre en cause l'approche fonctionnaliste et sociologique, largement inspirée des travaux de Christiane Nord et de la théorie du skopos (Reiss et Vermeer 1984), qu'a adoptée Mme Oittinen. De même qu'il est difficile d'ignorer les progrès des différentes théories de la réception en études littéraires. Dans le champ traductologique, on a également vu se déplacer le centre d'intérêt des recherches du texte original, considéré comme facteur d'altérité (Berman) à l'analyse des corpus de textes traduits (Venuti 2000, Baker 1998) en passant par la prise en compte des multiples lectures-interprétations (approche herméneutique). Comme le fait remarquer Venuti (2000), l'histoire de la théorie de la traduction peut être interprétée comme une relation mouvante entre l'autonomie du texte traduit (ou les actes du traducteur) et les concepts d'équivalence et de fonction. Il est vrai qu'après le succès du structuralisme, pour qui le texte était considéré comme objet stable et 
fermé, le débat théorique sur la traduction, dominé par le concept d'équivalence (de forme ou de sens), semblait inextricablement divisé. L'approche fonctionnelle, axée sur l'effet produit par la traduction dans la culture de réception, est alors apparue comme une solution à cette impasse théorique.

Il est indéniable que les facteurs sociologiques, historiques, culturels, le contexte de la réception (lecture du texte source par le traducteur et du texte traduit par son public cible), sont loin d'être négligeables dans l'analyse des traductions et qu'ils influent grandement sur l'acte même de la traduction. Il nous semble cependant que cette influence du contexte récepteur occupe une trop grande place dans l'analyse qu'en fait Riitta Oittinen: elle évacue en quelque sorte le texte de départ et le texte d'arrivée au profit des multiples interprétations de lecture, des projets traductifs et des conceptions diverses de l'éventuel lectorat. L'auteur s'appuie également sur les théories de l'esthétique de la réception, de l'herméneutique et du «reader oriented criticism» ou la déconstruction lorsque'elle affirme:

As a whole, the "rights» of the author of the original and the "rights » of the readers of the translation need not conflict; quite the contrary, authors have also thought of their future readers children - and have written, adapted, their own texts for them. Translators in turn complement, adapt the texts on the basis of their viewpoint of their own culture and language. When translating for children, taking into consideration the target-language children as readers is a sign of loyalty to the original author (p. 84).

Que ressort-il de tout cela? Toutes les interprétations sont-elles donc valables? Umberto Eco (1992: 41) a apporté des nuances éclairantes entre le lecteur empirique, le lecteur modèle, l'intentio operis et l'intentio auctoris:

L'initiative du lecteur consiste à émettre une conjecture sur l'intentio operis. L'ensemble du texte pris comme un tout organique - doit approuver cette conjecture interprétative, mais cela ne signifie pas que, sur un texte, il ne faille en émettre qu'une seule. Elles sont en principe infinies, mais à la fin, elles devront être testées sur la cohérence textuelle, laquelle désapprouvera les conjectures hasardeuses.

Autrement dit, les limites de l'interprétation coïncident avec les droits du texte.

Christiane Nord (1997: 124) a également formulé une critique semblable à propos de la théorie du skopos. En effet, le précepte selon lequel «the translation purpose justifies the translation procedures» est acceptable dans le cas où il y a correspondance entre l'objectif de la traduction et les intentions de l'auteur; mais qu'arrive-t-il dans le cas où la demande de traduction requiert une traduction dont les objectifs sont contraires ou incompatibles avec les opinions ou les intentions de l'auteur? «In this case, the Skopos rule could easily be interpreted as the end justifies the means, and there would be no restriction to the range of possible ends", conclut-elle et, devant ce risque de dérapage, elle avait invoqué le principe de loyauté:

Let me call «loyalty» this responsibility translators have toward their partners in translational interaction. Loyalty commits the translator bilaterally to the source and the target sides. It must not be mixed up with fidelity or faithfullness, concepts that usually refer to a relationship holding between the source and the target texts. Loyalty is an interpersonal category referring to a social relationship between people (p. 125).

Elle avait alors relevé (p. 112) une critique formulée par Werner Koller: les approches fonctionnelles transgressent les limites de la traduction proprement dite. En effet, en étendant l'objet d'étude de la traduction à la périphérie (le skopos), il y a risque de diluer les limites de l'acte traductif. Or Ritta Oittinen rejette les notions de «texte» et de «sens», qu'elle juge trop vagues, mais ne parvient pas à fonder son analyse sur des paramètres plus précis. Les notions d'intentions de l'auteur, d'images du lecteur récepteur de la traduction (l'horizon d'attente), d'infinité de lectures-interprétations de l'œuvre, à défaut de s'appuyer une théorie du langage, sont tout aussi vagues et subjectives. Comme l'a soulevé Venuti (2000: 5): 
The changing importance of a particular theoretical concept, whether autonomy, equivalence or function, may be determined by various factors, linguistic and literary, cultural and social.Yet, the most decisive determination is a particular theory of language or textuality.

À cet égard, nous croyons, à l'instar de Folkart (1991:14), qu'une approche basée sur la linguistique énonciative aurait été mieux adaptée à une analyse du matériau langagier qui tienne compte à la fois des données polysystémiques, des acteurs en présence et des transformations inhérentes à la traduction:

Réintroduire le sujet traduisant dans le texte traduit, faire la part de la subjectivité, reconnaitre la contingence dont est nécessairement grevée toute ré-énonciation, ce n'est nullement ouvrir les écluses aux débordements du n'importe comment. C'est, paradoxalement, le premier pas vers un plus grand respect du texte, du texte en situation d'énonciation, en interaction avec ses énonciataires, comme du texte en situation de ré-énonciation, en interaction avec son ré-énonciateur, avec ses ré-énonciataires.

Une telle approche aurait permis de traiter des composantes de l'acte traductif dans un cadre d'analyse plus rigoureux qui aurait mis en évidence, à l'intérieur du système global formé par le texte-source, le texte-cible et leurs cadres d'énonciation respectifs, les démarcatifs de nature à signaler le décalage énonciatif, le glissement de l'énonciation de l'auteur à celle du traducteur. Un inventaire des lieux qui manifestent, post factum, le débrayage-rembrayage qu'entraîne inéluctablement la traduction aurait également permis de raffiner les paramètres d'analyse de ces «démarcatifs qui attestent l'hybridisme essentiel du système traductionnel» (p. 130).

Elle aurait également permis d'apporter de plus nettes distinctions entre les différentes modalités d'expression (langagière, picturale, orale, musicale, etc.), les interprètes (traducteurs, lecteurs, enfants, illustrateurs, etc.) ainsi que les décalages (d'ordre référentiel, pragmatique et sémiologique) entraînés par la remédiation interlinguale. En effet, la tentation de couvrir l'ensemble des aspects de la traduction de la littérature pour enfants dans une approche alliant les concepts de la théorie bakhtinienne aux approches fonctionnaliste et sociologique nous semble relever d'une lecture un peu trop rapide en ce qui a trait aux conclusions qu'en tire l'auteur. Il en résulte une confusion théorique d'où il est difficile d'extraire des concepts définitoires clairs et rigoureux.

L'une des conséquences de ce choix théorique est que l'auteur en vient à traiter des aspects aussi différents que les acteurs de la traduction-communication, les données polysystémiques et fonctionnelles, les mediums langagier (le texte, les mots), oral (prosodie, rythme), pictural, et les attentes du public-cible, tout sur un même plan:

Translators of picture books translate whole situations including the words, the illustrations, and the whole (imagined) reading-aloud situation. Illustration is a many-faceted phenomenon in translation: on the one hand, illustrations go along with translations and their originals; on the other hand, illustrations can be understood as a form of translation as such. Yet I have deliberately avoided relying on any separate theory of illustrations and have dealt with illustration within the framework of translation, as part of the Bakhtinian dialogics of translation (p. 75).

Or, il nous semble injustifié de traiter le mode langagier et le mode pictural de l'illustration dans un cadre théorique aussi large que celui de la traduction en référence au concept de dialogisme et d'intertextualité de Bakhtine.

D'une part, les concepts de dialogisme et de polyphonie, développés par Bakhtine et approfondis plus tard par Julia Kristeva avec la notion d'intertextualité, ne font pas abstraction du texte: il faut entendre ce dialogue comme une modification radicale des relations (entre l'auteur, les personnages, l'espace, l'autre, le temps) mises en écriture, inscrites dans $l^{\prime}$ cuvre $^{5}$. C'est ainsi que se laisse percevoir ce travail dans la langue, cette signifiance: le «texte» n'est lui-même que par le «dialogisme» qui le définit.

D'autre part, si, comme le prétend Oittinen, les illustrations de contes pour enfants fonctionent au même titre que le verbal, nous croyons, comme l'avance Molinié (1998: 26- 
27), qu'il faut établir une distinction claire entre le fonctionnement de l'art verbal et celui des arts non verbaux:

On soutiendra $[\ldots]$ que seul le langage verbal élabore du sens, stricto sensu, et que, corollairement, le sémantique est le domaine spécifique et exclusif du langage verbal: [...] c'est la seule spécificité sémiotique du langage verbal $[\ldots]$. Les arts non-verbaux ne disent strictement rien $[\ldots]$. Ils indiquent $[\ldots]$. Ils font signe qu'il y a du monde [...].

Ainsi vaut-il mieux parler de sémiotique verbale, de sémiotique picturale ou de sémiotique musicale, puisqu'il s'agit de processus, de manifestations, de combinaisons et de réalisations de valeurs diverses. C'est le cas de la littérature pour enfants: les illustrations, tout en faisant partie intégrante de l'œuvre et en participant autant que le texte à la construction du sens, exigent une analyse sémiotique spécifique. Nous nous référons ici, à titre d'exemple, à l'étude d'un album pour enfants, effectuée par Nicole Éveraert-Desmedt (1990) selon les principes de la sémiotique peircienne. Elle montre, dans un cadre rigoureux, l'interaction des illustrations avec le texte: les images peuvent a) renvoyer à leur objet de façon iconique, sans nécessairement le représenter; b) mettre en relation le texte et l'image de façon indicielle («Voici»); ou c) de façon symbolique (en tant que signe d'un code sous-jacent). Nous croyons qu'un tel cadre aurait permis de mieux expliquer le caractère incongru ou étonnant de certaines illustrations que le recours à des notions aussi générales que le principe carnavalesque de Bakhtine.

Ainsi, à trop vouloir souligner l'influence des polysystèmes réceptifs (celui du traducteur-lecteur du texte original, et celui des lecteurs-cibles du texte traduit), l'auteur en vient à dénaturaliser le texte et, trop souvent, à s'en tenir à des pétitions de principes:

Loyalty implies respect for more than a text in words as such, or a certain form of content; it implies respect for an entire story-telling situation where a text is interpreted for new readers, who take the story as it is, who accept and reject, who react and respond. "Translation is a labour of love» (p. 83-84).

Le mérite principal de Mme Oittinen est sans doute d'avoir mis en évidence la complexité de la problématique de la traduction de la littérature pour enfants. Mais le sujet aurait mérité d'être traité dans un cadre méthodologique plus rigoureux, mettant à profit les théories du texte issues de la problématique de l'énonciation où l'approfondissement des concepts d'interdiscurivité et de translinguistique aurait davantage éclairé le sujet. L'ouvrage n'aidera pas nécessairement le traducteur praticien mais, par le foisonnement des concepts théoriques qu'il présente, il apporte une contribution certaine au domaine des recherches traductologiques. Nul doute que la question la littérature d'enfance et de jeunesse continuera à susciter un intérêt croissant: pensons au succès retentissant de livres tels Harry Potter ou The Lord of the Rings et au nombre de traductions, adaptations et études qu'ont déjà suscitées ces œuvres.

Louise Audet

Université de Montréal, Montréal, Canada

\section{NOTES}

1. $«[\ldots]$ as context - time, place and culture, including the individual interpreting the context and acting in the context.» (p. 9)

2. For Our Own Good (1990)

3. Poetics of Children Literature (1986)

4. Children's Fiction in the Hands of the Translator (1986)

5. En référence à Problèmes de l'œuvre de Dostoïevski, premier ouvrage de Bakhtine (1929) dans lequel il développe des concepts élaborés par le groupe Bakhtine, Medvedev et Volochinov, dans Peytard (1995). 


\section{RÉFÉRENCES}

BAKer, M. (1998) : «Réexplorer la langue de la traduction : une approche par corpus», Meta 434, p. $480-485$.

Eco, U. (1992): Les limites de l'interprétation, Paris, Grasset.

Éveraert-Desmedt, N. (1990): Le processus interprétatif, Mardaga, Liège.

Folkart, B. (1991): Le conflit des énonciations, Candiac (Québec), Balzac.

Klingberg, G. (1986): Children's Fiction in the Hands of the Translators, Studia psychologica et paedagogica, Series altera LXXXII, Lund, Bloms Boktryckeri Ab.

Miller, A. (1990a): For Your Own Good: Hidden Cruelty in Child Rearing and the Roots of Violence, New York, The Noonday Press.

_ (1990b): Banished Knowledge, New York, Double Day Press.

Molinié, G. (1998): Sémiostyslistique, Paris, Presses universitaires de France.

Nord, C. (1997): Translating as a Purposeful Activity. Functionalist Approaches Explained, Manchester, St Jerome Publishing.

Perrot, J. (1993): Actes du Xe Congrès de l'International Research Society for children's literature, Nancy, Presses Universitaires de Nancy.

Peytard, J. (1995) : Mikhaïl Bakhtine. Dialogisme et analyse du discours, Paris, Bertrand-Lacoste.

Reiss, K. and H. J. Vermeer (1984): Grundlegung einer allgemeinen Translationstheorie, Tübingen, Max Niemeyer Verlag.

Shavit, Z. (1986): Poetics of Children Literature, Athens, University of Georgia Press.

Venuti, L. (2000): The Translation Studies Reader, London/New York, Routledge.

\section{REMERCIEMENTS}

Je tiens à remercier Jeanne Dancette qui, par ses corrections et ses remarques toujours pertinentes, a beaucoup aidé à la révision de ce texte.

Percebois, J. (2002) : Terminologie anglais-français du commerce international: théories, politiques, accords et institutions, Paris, Economica, $296 \mathrm{p}$.

L'auteur, maitre de conférences d'anglais à la Faculté des sciences économiques de l'Université de Montpellier, a une longue expérience de l'enseignement de l'anglais économique. Son ouvrage "s'adresse à tous les étudiants dont le cursus inclut des références au commerce international, en particulier les [sic] étudiants des Facultés des Sciences Économiques, AES, IEP, Classes Préparatoires, Écoles supérieures de Commerce et IUT». Il s'inscrit dans le sillage des ouvrages précédents de l'auteur, dont il reprend la formule: L'anglais de la microéconomie et L'anglais de la macroéconomie, publiés chez le même éditeur.

Comment ce livre, présenté comme une terminologie du commerce international, se positionne-t-il par rapport aux nombreux et excellents dictionnaires et manuels d'économie bilingue?

Disons tout d'abord qu'il ne s'agit pas d'un dictionnaire, car il n'est pas organisé en termes. Il ne s'agit pas non plus d'un manuel d'anglais de spécialité, car il ne présente pas une étude de la langue du commerce international. Par sa facture, il ressemble plus à un cours d'économie puisqu'il est structuré autour des grands thèmes classiques du commerce international, comme l'annoncent tant le sous-titre; théories, politiques, accords et institutions, que la table des matières.

Ainsi, les deux premiers chapitres ont pour thèmes les théories consacrées au commerce international. Le troisième chapitre examine le libre-échange et le protectionnisme, puis aborde la question des barrières tarifaires et non tarifaires. Les accords internationaux et les institutions qui les régissent font l'objet du chapitre 4 . Le chapitre 5 traite des blocs 